\title{
Effects of nicotinic cholinergic system manipulations on paired-associate learning (PAL) in mice
}

\section{Melissa Milanovic}

Department of Psychology, College of Social and Applied Human Sciences, University of Guelph, Guelph, ON Canada. Faculty supervisor: Dr. Boyer Winters. For correspondence, please email: milanovmelissa@gmail.com.

\section{Abstract}

Rationale: The ability to perform on the Cambridge Neuropsychological Test Automated Battery touchscreen pairedassociate learning (PAL) test is predictive of Alzheimer's disease and Mild Cognitive Impairment. Recently, an automated computer touchscreen PAL task for mice has been developed. Pharmacological validation of this task is warranted to establish it as a useful tool in future drug discovery pertaining to Alzheimer's disease and Mild Cognitive Impairment.

Objectives: This investigation provides a systematic analysis of nicotinic involvement within the PAL task for mice. Particularly, the effects of systemic administration of nicotinic cholinergic agents (agonist and antagonist) on PAL task performance in C57BL/6 mice were investigated. This was done to detect whether bidirectional modification of performance is consequent upon these manipulations.

Methods: Upon acquiring the PAL task, nicotine (nicotinic receptor agonist; $0.1,0.5$, and $1.0 \mathrm{mg} / \mathrm{kg}$ ) and mecamylamine (nicotinic receptor antagonist; $0.3,1.0$, and $3.0 \mathrm{mg} / \mathrm{kg}$ ) were administered intraperitoneally to the mice in a withinsubjects design, prior to daily sessions in the PAL task.

Results: Nicotine did not have any significant effect on PAL performance improvement at any doses. However, mecamylamine did increase perseverative responding and reaction time in the mice. Such impairment effects are interpreted as being attentional in nature.

Conclusion: This investigation indicates that mice indeed acquire the rodent PAL task, deeming it a valuable tool for future drug discovery. Further, the nicotinic cholinergic system appears to be implicated in PAL task performance, with greater effects seen with deactivation rather than activation of the system, and with these effects appearing to be of an attentional nature.

Keywords: paired-associate learning (PAL); Alzheimer's disease; nicotinic cholingeric system; touchscreen

\section{Introduction}

Animal models in research have been instrumental in expanding the understanding of the biological bases of behavior. Many fundamental issues in the field of psychology have been examined using animal subjects, including the functional mechanisms that influence learning and memory, and the psychopharmacological implications associated with these mechanisms (Domjan and Purdy 1995). Particularly significant are rodent models which, from their performance on behavioral tasks, provide results with potential significance to human patients with conditions that affect these behaviors (Bartko et al. 2011). One such translatable model that pertains to human conditions such as Alzheimer's disease and Mild Cognitive Impairment is a fundamental component of the present investigation.

\section{Human PAL research}

onist; $0.1,0.5$, and $1.0 \mathrm{mg} / \mathrm{kg}$ ) and mecamylamine

A computerized test battery, the Cambridge Neuropsychological Test Automated Battery (CANTAB) was designed with a focus on neuropsychological functions subserved by the frontostriatal circuitry (Fray et al. 1997). These circuits consist of neural pathways that connect the frontal lobe brain regions to different sectors of the striatum, which mediate cognitive, behavioural and motor programs within the brain (Alexander et al. 1986). Included in this test battery is a paired-associate learning (PAL) test, which provides a useful measure for assessing human patients with Mild Cognitive Impairment or potential Alzheimer's disease. In this test, participants are faced with boxes presented on a 


\section{Effects of nicotinic cholinergic system manipulations of PAL in mice (Milanovic)}

computer screen; the boxes were opened in a random order, revealing distinctive visual patterns or 'objects' in each location on the screen. Once the boxes have been opened, the participants are presented each visual pattern one-by-one, in the middle of the screen and they must touch the box in which they had seen it located. Essentially, this is a visuospatial task whereby the test-taker must learn to associate a visual pattern with a particular spatial location in which it was presented.

It is known that visual representations of objects are processed predominately within the perirhinal cortex (Mumby and Pinel 1994; Winters and Bussey 2005), whereas spatial information is processed in the hippocampus (O'Keefe and Nadel 1978; Aggleton et al. 1986; Forwood et al. 2005). These areas are primarily affected by Alzheimer's disease, which is a progressive brain disease characterized by memory and cognitive impairment (Braak and Braak 1997). As such, the CANTAB PAL test provides a measure that is sensitive to the function (and functional interaction) of areas implicated in the cognitive impairments associated with Alzheimer's disease and patients indeed demonstrate robust deficits in this task (Junkkila et al. 2012). Furthermore, previous investigation into the sensitivity of the CANTAB PAL task identified PAL performance as an informative diagnostic tool. Particularly, these investigations determined PAL performance to be an effective measure in discerning the impairments as seen in the early stages of Alzheimer's disease from the cognitive impairment associated with separate conditions (Sahakian et al. 1989; Swainson et al. 2001; Blackwell et al. 2004).

\section{Animal models of PAL}

While having the CANTAB PAL test that is administrable to human participants is indispensable, it is also valuable to have animal models that parallel this task, which enables for pharmacological testing and research into implicated biological systems. Variants of the PAL task have been developed for research on rats (Talpos et al. 2009), monkeys (Katner et al. 2004), and pigeons (Rodewald 1973). However, a separate rodent, the mouse, is becoming increasingly used in neurobiological research. A particular usefulness of mice is the various transgenic and knockout strains, which have enhanced experimental pathology by fostering dramatic advances in the understanding of the pathogenesis of many human diseases (Aguzzi et al. 1994). In the context of the present research, a task designed for mice that parallels the CANTAB PAL would provide the ability to behaviourally phenotype various transgenic and knockout mice. Having acknowledged the need for a mouse model to be used within PAL research, Bussey et al. (2001) demonstrated using a computer-automated touchscreen method that mice are indeed capable of complex visual discriminations, as had been seen on similar tests of rats, monkeys and humans. Bartko et al. (2011) investigated this computer-automated touchscreen method of PAL in mice, providing evidence that mice could indeed acquire the rodent
PAL task and thus expanding the subject pool for PAL research to include the experimental mouse. Furthermore, Bartko and al. (2011) also examined the effect of manipulations of the muscarinic cholinergic system via administration of various muscarinic antagonists, as well as the effects of cholinesterase inhibitor drugs. The study demonstrated the importance of the muscarinic cholinergic system in mediating PAL performance, as the muscarinic receptor antagonists scopolamine and dicyclomine impaired accuracy of mice in the PAL task. The study provided pharmacological validation of this PAL task due to the fact that muscarinic receptor antagonism, a manipulation that consistently disrupts many aspects of cognition (Drachman and Leavitt 1974; Hagan et al. 1987; Bartholomew et al. 2009), impaired task performance. This indicated that the muscarinic cholinergic system is influential in PAL task ability, and as such, this task was demonstrated as a tool of considerable value for further drug discovery.

\section{The role of the cholinergic system in cognition}

Acetylcholine is a neurotransmitter that acts on two main classes of receptors. This includes the nicotinic cholinergic receptors, which are ionotropic and therefore ligand-gated ion channels that become activated upon the binding of acetylcholine and other various nicotinic agonists such as nicotine (Graef et al. 2011). Muscarinic or metabotropic cholinergic receptors are the second class of receptors, comprised of G-protein-coupled receptors that when activated through the binding of acetylcholine and other various muscarinic agonists (such as muscarine) results in transmission of signals inside the cell by an intracellular second messenger system (Caulfield 1993).

The role of the cholinergic system in cognition has been extensively studied and particularly relevant to the present investigation are various findings that have implicated acetylcholine in the cognitive dysfunction of Alzheimer's disease. Experimental analyses completed in both humans and non-human primates have demonstrated that memory impairments similar to those seen in aged individuals with Alzheimer's disease are inducible in young subjects by administration of the muscarinic antagonist scopolamine, which blocks central cholinergic activity (Bartus and Johnson 1976; Frumin et al. 1976; Drachman 1977). Additionally, administration of the cholinergic agonist physostigmine, which prevents the degradation of acetylcholine, reversed the scopolamine-induced cognitive impairment in these subjects (Drachman and Leavitt 1974; Drachman 1977; Bartus 1978). Additional demonstration of a role of the muscarinic cholinergic system in cognition as related to Alzheimer's disease was provided by the work of Bartko et al. (2011), showing an importance in mice of the muscarinic cholinergic system in the rodent model of the Alzheimer's disease-sensitive PAL task as described above.

Unlike the muscarinic system, the nicotinic system has yet to be comprehensively studied in any animal model for its involvement in the PAL task. As discussed, the ability to 


\section{Effects of nicotinic cholinergic system manipulations of PAL in mice (Milanovic)}

learn paired-associates is sensitive to cognitive deficits in patients with Alzheimer's disease, as proven by the poor performance of these individuals on the CANTAB PAL (Fray et al. 1997). The nicotinic cholinergic mechanisms have been considered important in explaining the pathophysiology of and designing treatments for Alzheimer's disease, due to an observation that these patients have reduced cortical nicotinic cholinergic receptor binding compared to age-matched healthy individuals (James and Nordberg 1995). As such, the nicotinic cholinergic system might have relevance to performance in the PAL task. The above facts, in addition to the existence of a recently validated mouse model, provided the opportunity and impetus to investigate the nicotinic cholinergic system, with the intent of providing further pharmacological validation of this PAL task for mice.

An additional role of the present study has been to help elucidate an outcome of ambiguous nature from the investigation into PAL in mice by Bartko et al. (2011). In particular, they had discovered that administration of donepezil (a common drug used in the treatment of Alzheimer's disease-type dementia in humans) improved PAL performance. As an acetylcholinesterase inhibitor, this effect of donepezil could have implications for both the muscarinic and nicotinic receptor functions. By providing a comprehensive investigation into the role of nicotinic receptors in PAL performance, the present investigation should help to clarify whether increased nicotinic receptor activation might have contributed to the improved performance in PAL as had been seen following administration of donepezil.

\section{Nicotinic cholinergic receptors}

Recent relevant research into the role of nicotinic receptors in learning and memory has demonstrated that administration of the nicotinic receptor agonist nicotine enhances object memory in rats via nicotinic receptor activation in the perirhinal cortex and hippocampus (Melichercik et al. 2012). Levin et al. (2006) have also discussed various memory improvements following nicotine administration, including improved working memory, enhanced performance of spatial discrimination tasks and elevated object recognition in rats. Katner et al. (2004) provided complementary evidence that visuospatial associative memory is impaired by deactivating the nicotinic system through administration of the nicotinic antagonist drug mecamylamine in rhesus monkeys. Additionally, mecamylamine has been demonstrated to reduce spatial working memory performance in rats (Kim and Levin 1996). These findings support a role for the nicotinic system in memory and highlight the lack of PAL research within a mouse model. As such, the present study sought to assess the effects of nicotinic receptor agonism and antagonism on PAL task performance in mice.

Despite the limited amount of PAL research to date, within the domains of attention and cognition, nicotinic effects have been more commonly researched. Several studies have described enhanced performance of mice in the 5-Choice Serial Reaction Time Task (5CSRTT) adapted by Carli et al. (1983). As a measure of attentional performance, animals must monitor an array of five screens and respond to a stimulus randomly presented briefly in one of them. The spatial distribution of attention across all five screens places demands on divided attention to perform visual search, and as such, is an appropriate task of attention (Hahn et al. 2002). Administration of nicotine in mice has been shown to improve attention on this task in comparison to mice treated with vehicle (Young et al. 2004; de Bruin et al. 2006). Such findings may be relevant to the present investigation, because should an effect of nicotine be seen in the PAL performance of mice, the nature of the effect will need to be considered (i.e. whether the effect is attentional or memory-based).

\section{Objectives}

The utility and clinical significance of a mouse version of the PAL task has been demonstrated via manipulation of the muscarinic cholinergic system in previous work (Bartko et al. 2011). Since mice are an integral component in testing therapeutic agents and researching principles relevant to human cognitive disorders, having this PAL task extensively validated will help to establish it as a useful tool for research into pharmacological and biological components of cognition. Furthermore, since PAL is known to be impaired in patients with Alzheimer's disease (Fray et al. 1997), the particular systems implicated in this cognitive disorder can be studied quite directly using a PAL task.

In the present investigation, the cholinergic system was studied, and particularly, manipulation of the nicotinic cholinergic system receptors was assessed for their impact on PAL performance, thereby furthering the utility of this task. Nicotine (a nicotinic receptor agonist) and mecamylamine (a nicotinic receptor antagonist) were investigated for their drug effects on PAL performance in mice. An effect of bidirectional modification was anticipated, such that nicotine would enhance PAL performance while mecamylamine would impair PAL performance. A demonstration of this effect would provide evidence to support the fundamentality of the nicotinic system in paired-associate learning; essentially, should an agonist of the nicotinic receptors enhance PAL performance and an antagonist of the nicotinic receptors impair PAL performance, then this would indicate that the nicotinic system is needed to guide behaviour on the basis of visuospatial associations. Thus, the research question proposed for the present investigation was: using a mouse model of PAL, would manipulation of nicotinic cholinergic receptors via antagonism and agonism show bidirectional modification on performance in the PAL task? 


\section{Methods}

\section{Subjects}

Subjects were seven C57BL/6 male mice (Charles River Laboratories, QC, Canada) aged two months old at the beginning of testing. See Supplementary Information for additional description of subjects.

\section{Apparatus}

Testing was conducted in a touchscreen-based automated operant system for mice (Bussey et al. 2001; Morton et al. 2006). The operant box contained an infrared touchscreen $(16.0 \mathrm{~cm}$ high $\times 21.2 \mathrm{~cm}$ wide $)$ at the end opposite the magazine. The touchscreen used infrared photocells; thus the mouse was not required to exert pressure on the screen for the nose-poke to be detected. A black Perspex mask (11.80 $\mathrm{cm}$ high $\times 22.8 \mathrm{~cm}$ wide) with three response windows (5.80 $\mathrm{cm}$ high $\times 5.0 \mathrm{~cm}$ wide) was placed over the screen, through which the mouse was able to make a nose-poke towards any of the three screen locations.

\section{Pretraining}

A pretraining protocol was followed whereby the mice were familiarized with the operant chamber, visual training stimuli and the collection of milkshake upon making nosepoke responses. Refer to Supplementary Information for the daily pretraining procedure.

\section{PAL task training and criterion levels}

Upon successful completion of pretraining, mice were trained on the PAL task. Refer to Supplementary Information for this training procedure.

Various levels of accuracy criterion were selected to which the mice were required to reach, prior to each drug study. Drug study A consisted of analysis of the effects of nicotine on PAL performance. Bartko et al. (2011), having been the only other researchers to investigate this PAL task in mice, trained their mice to $80 \%$ PAL task accuracy prior to drug study commencement. Their investigation into facilitation effects of donepezil (proposed to enhance performance) established only the highest dose administered to be significant in improving PAL performance. As such, it was decided to train the mice to a criterion of $70 \%$ accuracy for drug study A. This provided a larger gap between criterion (70\%) and the highest possible performance $(100 \%)$, allowing for better detection of nicotinic agonist facilitation effects. Thus, the mice needed to complete all 36 trials with $70 \%$ accuracy over three consecutive days. Upon reaching this criterion, the training of these mice was halted until all mice in the group reached the $70 \%$ accuracy. Once all mice reached criterion, they were re-baselined on the task for six days to stabilize performance. On three of these rebaseline days, each mouse received an intraperitoneal injection of saline (a mock injection) 20 minutes prior to entry in the PAL task session. This was done as an adjustment process, to allow the mice to get accustomed to the injection procedure that would be used in the pharmacological investigations. Following this, drug study A (nicotine) began.

Drug study B consisted of examining the effects of mecamylamine on PAL performance in the same group of mice. Since antagonism of the nicotinic system was proposed to impair performance, the mice were trained to a higher criterion upon completion of drug study A. As such, they needed to complete all 36 trials with $80 \%$ accuracy over three consecutive days. This was done to allow for a wider gap between chance accuracy (50\%) and trained accuracy $(80 \%)$, to better detect an impairment effect of mecamylamine.

Drug study $\mathrm{C}$ consisted of examining the effects of nicotine in these mice a second time while the mice were still at an $80 \%$ accuracy level post-drug study B. This was done to provide a more thorough and comprehensive analysis of nicotine on potential improvement of PAL performance, which was consistent with what Bartko et al. (2011) had done to examine improvement effects.

Between each of the three drug studies, the mice underwent re-baselining on the PAL task for seven days and received a mock infusion of saline prior to two of these rebaselining sessions to keep them accustomed to the injection procedure. Between drug study $\mathrm{A}$ and $\mathrm{B}$, additional training sessions were required to raise their accuracy level from $70 \%$ to $80 \%$. Four dosages were determined and assessed within the mice for each drug being studied. During all drug studies, one washout day was used between treatments. On these days the mice were re-baselined on the PAL task (i.e. completed a session in the PAL task without any injection procedure).

\section{Pharmacological experiments}

Nicotine and mecamylamine were administered intraperitoneally with injection volumes of $0.2 \mathrm{ml}$ per $20 \mathrm{~g}$ of the weight of the mouse. Drugs were dissolved in physiological saline ( $0.9 \%$ sodium chloride, $\mathrm{pH} 7.0)$ to create appropriate stock solutions. A Latin square design was used to determine the sequence of drug administrations for each mouse for each individual drug study, resulting in a withinsubjects model for each drug investigation. For each injection procedure, the drug was administered 20 minutes prior to the initiation of the PAL session.

In drug study $\mathrm{A}$, the effects of nicotine on PAL performance were examined. Nicotine was administered to mice having reached $70 \%$ accuracy on the PAL task at three doses $(0.1,0.5$, and $1.0 \mathrm{mg} / \mathrm{kg})$, with a fourth dose condition of saline (to serve as a vehicle control condition). These doses have been shown to be effective in studies of nicotine effects on other learning and memory tasks, with particularly 0.1 and $0.5 \mathrm{mg} / \mathrm{kg}$ of nicotine having resulted in improved memory-related effects in mice when used on assessment in the elevated plus maze (Biala and Kruk 2008). 


\section{Effects of nicotinic cholinergic system manipulations of PAL in mice (Milanovic)}

In drug study $B$, the effects of mecamylamine administration were examined in mice having reached $80 \%$ accuracy on the PAL task. Mecamylamine was given at three doses $(0.3,1.0$, and $3.0 \mathrm{mg} / \mathrm{kg})$, with a fourth dose condition of saline. Literature indicates that doses of mecamylamine reported to impair memory in mice models have ranged from $0.1 \mathrm{mg} / \mathrm{kg}$ to $25.0 \mathrm{mg} / \mathrm{kg}$ (Terry et al. 1999), with doses below $1.0 \mathrm{mg} / \mathrm{kg}$ being regarded as moderately selective at antagonizing nicotinic receptors (Clarke et al. 1994). As such, $1.0 \mathrm{mg} / \mathrm{kg}$, as well as a dose below $(0.3 \mathrm{mg} / \mathrm{kg})$ and above $(3.0 \mathrm{mg} / \mathrm{kg})$ were selected.

In drug study $\mathrm{C}$, the effects of nicotine administration on PAL performance were examined an additional time, while the mice were at an $80 \%$ trained accuracy post-drug study B. Nicotine was given at the same doses as in drug study A (0.1, 0.5 , and $1.0 \mathrm{mg} / \mathrm{kg}$ ), as well as a saline control.

\section{Data analysis}

Group means of accuracy (percent correct) were analyzed for each drug study to assess the effects of the nicotinic drugs on PAL performance. In addition, group means of reaction time (the length of time between a nose-poke to the magazine at the back of the chamber and a nose-poke to the touchscreen) for both correct and incorrect trials were analyzed, providing a measure of attentional effects of the drugs. Furthermore, group means of magazine latency (the length of time between a correct choice nose-poke to the touch screen and a nose-poke for food in magazine) were analyzed. Finally, group means of the total number of correction trials committed during each drug study were analyzed to investigate whether the drugs had a perseverative effect on mice in PAL. Means were submitted to one-way ANOVA with repeated measures for drug. Paired sample ttest comparisons were used for post hoc analyses of withinsubject effects of drug. All statistical analyses were conducted with a significance level of $\mathrm{p}=0.05$.

\section{Results}

\section{Drug study $A$}

Nicotine was administered systemically at three dose levels $(0.1 \mathrm{mg} / \mathrm{kg}, 0.5 \mathrm{mg} / \mathrm{kg}$, and $1.0 \mathrm{mg} / \mathrm{kg})$ and was predicted to produce an improvement in PAL task performance relative to the saline control condition.

Percent Correct (Accuracy). There was no overall effect of the drug on accuracy following administration of nicotine in the PAL task $\left(\mathrm{F}_{(3,18)}=0.031, \mathrm{p}=0.992\right.$; see Table 1 for mean accuracies \pm SEM).

Reaction Time. Reaction times for correct trials $\left(\mathrm{F}_{(1.062,6.374)}=1.827, \mathrm{p}=0.224\right)$ and incorrect trials $\left(\mathrm{F}_{(1.786,10.719)}=1.981, \mathrm{p}=0.187\right)$ were not significantly affected by nicotine (see Table 1 for mean reaction times \pm SEM for the correct trials and incorrect trials).
Magazine Latency. Magazine latency was not significantly affected by administration of nicotine $\left(\mathrm{F}_{(1.212,7.270)}=0.624, \mathrm{p}=0.485\right.$; see Table $\mathbf{1}$ for mean magazine latencies \pm SEM).

Correction Trials. Administration of nicotine did not significantly affect the number of correction trials committed by mice in the PAL task $\left(\mathrm{F}_{(3,18)}=0.904, \mathrm{p}=0.459\right.$; see Table 1 for mean number of correction trials \pm SEM).

\section{Drug study B}

Mecamylamine, a nicotinic receptor antagonist was administered systemically at three dose levels $(0.3 \mathrm{mg} / \mathrm{kg}$, $1.0 \mathrm{mg} / \mathrm{kg}$, and $3.0 \mathrm{mg} / \mathrm{kg}$ ) and a fourth saline condition, and was predicted to have an impairment effect on PAL task performance.

Percent Correct (Accuracy). Administration of mecamylamine did not have a significant effect on accuracy in the PAL task $\left(\mathrm{F}_{(3,18)}=0.912, \mathrm{p}=0.455\right.$; see Table $2 \mathrm{~A}$ for mean accuracies \pm SEM).

Reaction Time. Reaction time for correct trials $\left(\mathrm{F}_{(1.043,6.259)}=5.984, \mathrm{p}=0.048\right)$ and incorrect trials $\left(\mathrm{F}_{(1.377,8.263)}=11.026, \mathrm{p}=0.007\right)$ were both significantly affected by mecamylamine (see Table $\mathbf{2 A}$ for mean reaction times \pm SEM for correct and incorrect reaction times, Table 2B for post hoc paired-samples t-test comparisons and Figures 1 and 2 for graphs of each).

Magazine Latency. Magazine latency for correct trials was significantly affected by injections of mecamylamine $\left(\mathrm{F}_{(3,18)}=9.170, \mathrm{p}=0.001\right.$; see Table 2A for mean magazine latencies \pm SEM and Table $2 \mathbf{B}$ for post hoc paired-samples ttest comparisons).

Correction Trials. Administration of mecamylamine significantly affected the number of correction trials committed by the mice in the PAL task $\left(\mathrm{F}_{(3,18)}=3.419\right.$, $\mathrm{p}=0.040$; see Table $\mathbf{2 A}$ for mean correction trials \pm SEM, Table 2B for post hoc paired-samples t-test comparisons, and Figure 3 for graph).

\section{Drug study C}

The effect of nicotine was assessed a second time, to complement the procedure followed by Bartko et al. (2011), commencing with the mice having attained $80 \%$ accuracy (a higher criterion than used for the assessment of nicotine in drug study A). In this study, seizure activity occurred in all mice at the highest dose of nicotine administered (1.0 $\mathrm{mg} / \mathrm{kg}$ ). This was presumably due to downregulation of nicotinic receptors as a consequence of exposure to nicotine in drug study A. As such, analyses were conducted on performance at nicotine doses of $0.5 \mathrm{mg} / \mathrm{kg}$ and $0.1 \mathrm{mg} / \mathrm{kg}$, as well as saline.

Percent Correct (Accuracy). There was no overall effect of nicotine on PAL task accuracy $\left(\mathrm{F}_{(2,10)}=0.716, \mathrm{p}=0.512\right.$; see Table 3A for mean accuracies \pm SEM). 


\section{Effects of nicotinic cholinergic system manipulations of PAL in mice (Milanovic)}

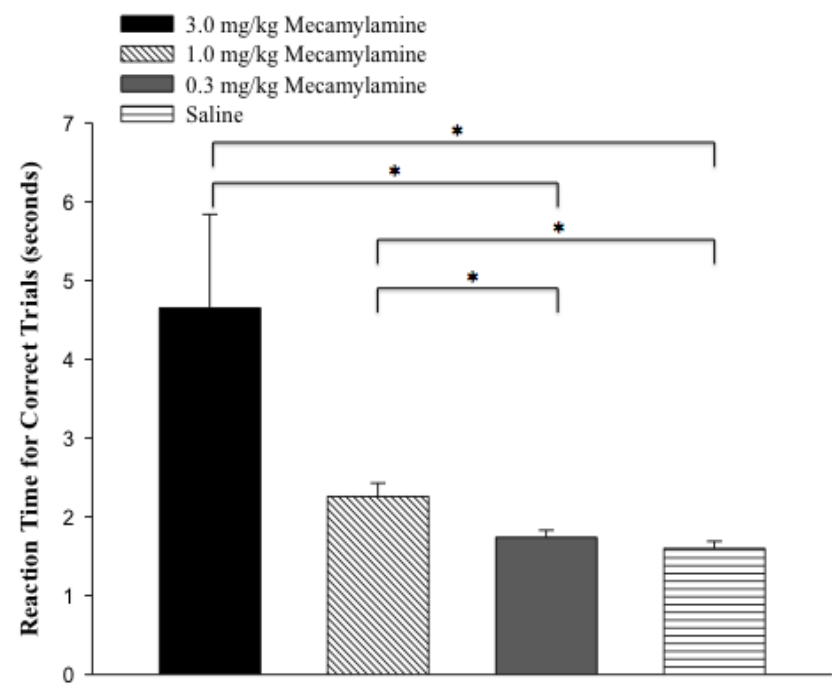

Figure 1. Significant effect of mecamylamine on reaction time for correct trials. Data are presented as mean reaction time for correct trials \pm SEM, with paired-samples significance indicated.

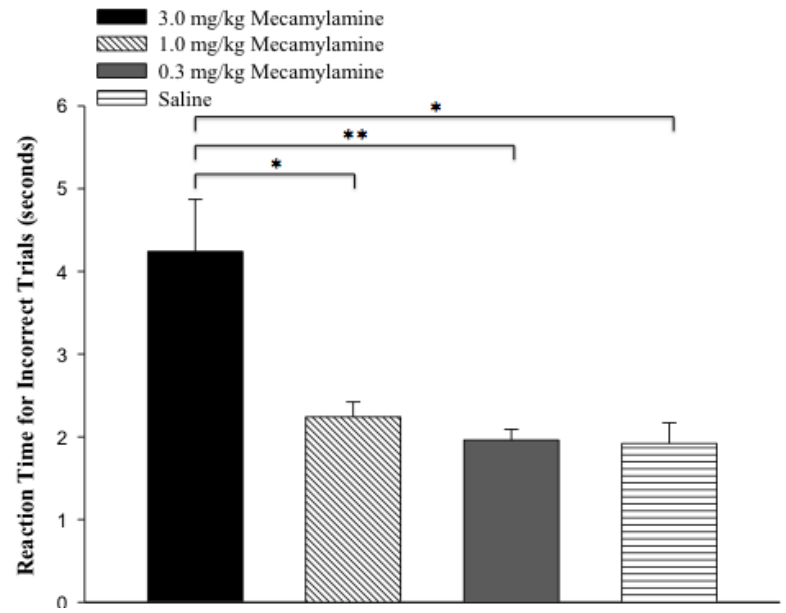

Figure 2. Significant effect of mecamylamine on reaction time for incorrect trials. Data are presented as mean reaction time for correct trials \pm SEM, with paired-samples significance indicated.

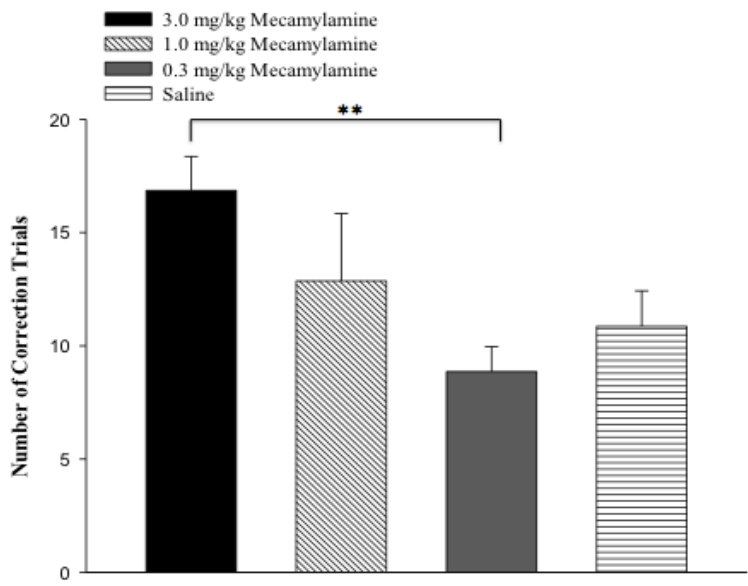

Figure 3. Significant effect of mecamylamine on number of correction trials. Data are presented as mean number of correction trials \pm SEM, with pairedsamples significance indicated. 


\section{Effects of nicotinic cholinergic system manipulations of PAL in mice (Milanovic)}

Table 1. Results of the nicotine drug study (drug study A), showing means of accuracy, reaction time for correct and incorrect trials, magazine latency, and committed correction trials. Values are provided as mean \pm SEM.

\begin{tabular}{|c|c|c|c|c|}
\hline & $1.0 \mathrm{mg} / \mathrm{kg}$ Nicotine & $0.5 \mathrm{mg} / \mathrm{kg}$ Nicotine & $0.1 \mathrm{mg} / \mathrm{kg}$ Nicotine & Saline \\
\hline Percent Correct (Accuracy) & $74.80 \pm 4.14$ & $74.21 \pm 4.40$ & $73.65 \pm 3.68$ & $73.69 \pm 2.17$ \\
\hline RT Correct Trials & $6.11 \pm 2.75$ & $2.80 \pm 0.63$ & $2.22 \pm 0.22$ & $2.44 \pm 0.58$ \\
\hline RT Incorrect Trials & $10.45 \pm 4.06$ & $2.78 \pm 0.58$ & $2.08 \pm 0.18$ & $5.48 \pm 3.68$ \\
\hline Magazine Latency & $1.24 \pm 0.08$ & $1.41 \pm 0.45$ & $1.07 \pm 0.06$ & $2.19 \pm 1.15$ \\
\hline Number of Correction Trials & $10.17 \pm 1.98$ & $14.43 \pm 3.23$ & $13.00 \pm 2.10$ & $14.86 \pm 2.30$ \\
\hline
\end{tabular}

Table 2A. Results of the mecamylamine drug study (drug study B), showing means of accuracy, reaction time for correct and incorrect trials, magazine latency, and committed correction trials. Values are provided as mean \pm SEM.

\begin{tabular}{|c|c|c|c|c|}
\hline & $3.0 \mathrm{mg} / \mathrm{kg}$ Mecamylamine & $1.0 \mathrm{mg} / \mathrm{kg}$ Mecamylamine & $0.3 \mathrm{mg} / \mathrm{kg}$ Mecamylamine & Saline \\
\hline Percent Correct (Accuracy) & $73.76 \pm 1.90$ & $77.78 \pm 4.50$ & $79.76 \pm 2.40$ & $79.37 \pm 2.90$ \\
\hline RT Correct Trials & $4.65 \pm 1.19$ & $2.26 \pm 0.17$ & $1.74 \pm 0.09$ & $1.60 \pm 0.09$ \\
\hline RT Incorrect Trials & $4.24 \pm 0.63$ & $2.24 \pm 0.18$ & $1.96 \pm 0.13$ & $1.92 \pm 0.25$ \\
\hline Magazine Latency & $1.46 \pm 0.06$ & $1.29 \pm 0.08$ & $1.04 \pm 0.07$ & $1.09 \pm 0.10$ \\
\hline Number of Correction Trials & $16.86 \pm 1.50$ & $12.86 \pm 2.99$ & $8.86 \pm 1.10$ & $10.86 \pm 1.57$ \\
\hline
\end{tabular}

Table 2B. Paired-samples $t$-tests of mean reaction time for correct and incorrect trials, magazine latency, and number of correction trials for the mecamylamine drug study.

\begin{tabular}{|c|c|}
\hline RT Correct Trials & Paired Sample t-tests \\
\hline $3.0 \mathrm{mg} / \mathrm{kg}-1.0 \mathrm{mg} / \mathrm{kg}$ & $t_{(6)}=2.068(p=.084)$ \\
\hline $3.0 \mathrm{mg} / \mathrm{kg}-0.3 \mathrm{mg} / \mathrm{kg}$ & $t_{(6)}=2.481(p=.048)^{*}$ \\
\hline $3.0 \mathrm{mg} / \mathrm{kg}$ - Saline & $t_{(6)}=2.711(p=.035)^{*}$ \\
\hline $1.0 \mathrm{mg} / \mathrm{kg}-0.3 \mathrm{mg} / \mathrm{kg}$ & $\mathrm{t}_{(6)}=3.375(p=.015)^{*}$ \\
\hline $1.0 \mathrm{mg} / \mathrm{kg}$ - Saline & $t_{(6)}=3.664(p=.011)^{*}$ \\
\hline $0.3 \mathrm{mg} / \mathrm{kg}$ - Saline & $t_{(6)}=1.278(p=.248)$ \\
\hline RT Incorrect Trials & Paired Sample t-tests \\
\hline $3.0 \mathrm{mg} / \mathrm{kg}-1.0 \mathrm{mg} / \mathrm{kg}$ & $t_{(6)}=3.133(p=.020)^{*}$ \\
\hline $3.0 \mathrm{mg} / \mathrm{kg}-0.3 \mathrm{mg} / \mathrm{kg}$ & $t_{(6)}=3.911(p=.008)^{\star \star}$ \\
\hline $3.0 \mathrm{mg} / \mathrm{kg}$ - Saline & $t_{(6)}=3.557(p=.012)^{*}$ \\
\hline $1.0 \mathrm{mg} / \mathrm{kg}-0.3 \mathrm{mg} / \mathrm{kg}$ & $\mathrm{t}_{(6)}=1.321(\mathrm{p}=.235)$ \\
\hline $1.0 \mathrm{mg} / \mathrm{kg}$ - Saline & $t_{(6)}=1.022(p=.346)$ \\
\hline $0.3 \mathrm{mg} / \mathrm{kg}$ - Saline & $\mathrm{t}_{(6)}=.226(\mathrm{p}=.828)$ \\
\hline
\end{tabular}

\begin{tabular}{|c|c|}
\hline Magazine Latency & Paired Sample t-tests \\
\hline $3.0 \mathrm{mg} / \mathrm{kg}-1.0 \mathrm{mg} / \mathrm{kg}$ & $\mathrm{t}_{(6)}=3.013(\mathrm{p}=.024)^{*}$ \\
\hline $3.0 \mathrm{mg} / \mathrm{kg}-0.3 \mathrm{mg} / \mathrm{kg}$ & $t_{(6)}=8.719(p=.000)^{\star \star *}$ \\
\hline $3.0 \mathrm{mg} / \mathrm{kg}$ - Saline & $t_{(6)}=3.889(p=.008)^{* *}$ \\
\hline $1.0 \mathrm{mg} / \mathrm{kg}-0.3 \mathrm{mg} / \mathrm{kg}$ & $t_{(6)}=3.281(p=.017)^{*}$ \\
\hline $1.0 \mathrm{mg} / \mathrm{kg}$ - Saline & $t_{(6)}=1.660(p=.148)$ \\
\hline $0.3 \mathrm{mg} / \mathrm{kg}$ - Saline & $\mathrm{t}_{(6)}=-.397(\mathrm{p}=.705)$ \\
\hline Correction Trials & Paired Sample t-tests \\
\hline $3.0 \mathrm{mg} / \mathrm{kg}-1.0 \mathrm{mg} / \mathrm{kg}$ & $t_{(6)}=1.316(p=.236)$ \\
\hline $3.0 \mathrm{mg} / \mathrm{kg}-0.3 \mathrm{mg} / \mathrm{kg}$ & $t_{(6)}=4.943(p=.003)^{\star *}$ \\
\hline $3.0 \mathrm{mg} / \mathrm{kg}$ - Saline & $t_{(6)}=2.349(p=.057)$ \\
\hline $1.0 \mathrm{mg} / \mathrm{kg}-0.3 \mathrm{mg} / \mathrm{kg}$ & $\mathrm{t}_{(6)}=1.280(\mathrm{p}=.248)$ \\
\hline $1.0 \mathrm{mg} / \mathrm{kg}$ - Saline & $\mathrm{t}_{(6)}=.718(p=.500)$ \\
\hline $0.3 \mathrm{mg} / \mathrm{kg}$ - Saline & $\mathrm{t}_{(6)}=-.890(p=.408)$ \\
\hline
\end{tabular}

Table 3A. Results of the second nicotine drug study (drug study C), showing means of accuracy, reaction time for correct and incorrect trials, magazine latency, and committed correction trials. Values are provided as mean \pm SEM.

\begin{tabular}{lccc}
\hline & $\mathbf{0 . 5} \mathbf{~ m g} / \mathbf{k g}$ Nicotine & $\mathbf{0 . 1} \mathbf{~ m g} / \mathbf{k g}$ Nicotine & Saline \\
\hline Percent Correct (Accuracy) & $81.02 \pm 2.20$ & $83.33 \pm 3.80$ & $86.11 \pm 1.90$ \\
RT Correct Trials & $1.80 \pm 0.19$ & $1.61 \pm 0.12$ & $1.45 \pm 0.09$ \\
RT Incorrect Trials & $1.83 \pm 0.30$ & $1.54 \pm 0.24$ & $1.88 \pm 0.09$ \\
Magazine Latency & $1.05 \pm 0.07$ & $0.99 \pm 0.07$ & $0.98 \pm 0.04$ \\
Number of Correction Trials & $10.50 \pm 2.41$ & $7.17 \pm 1.47$ & $6.33 \pm 1.12$ \\
\hline
\end{tabular}

Table 3B. Paired-samples $t$-tests of mean reaction time for correct trials.

\begin{tabular}{ll}
\hline RT Correct Trials & Paired-Sample t-tests \\
\hline $0.5 \mathrm{mg} / \mathrm{kg}-\mathbf{0 . 1} \mathrm{mg} / \mathrm{kg}$ & $\mathrm{t}_{(5)}=2.304(\mathrm{p}=.069)$ \\
$\mathbf{0 . 5} \mathrm{mg} / \mathrm{kg}$ - Saline & $\mathrm{t}_{(5)}=\mathbf{2 . 8 3 4}(\mathrm{p}=.037)^{\star}$ \\
$\mathbf{0 . 1} \mathrm{mg} / \mathrm{kg}$ - Saline & $\mathrm{t}_{(5)}=-2.180(\mathrm{p}=.081)$ \\
\hline
\end{tabular}


Reaction Time. There was a significant effect of nicotine on reaction time for correct trials $\left(\mathrm{F}_{(2,10)}=6.713, \mathrm{p}=0.014\right)$, but not for incorrect trials $\left(\mathrm{F}_{(2,10)}=1.811, \mathrm{p}=0.213\right.$; see Table 3A for mean reaction times \pm SEM for correct and incorrect reaction times and Table 3B for post-hoc pairedsamples t-test comparisons between dose conditions for reaction time on correct trials).

Magazine Latency. Magazine latency was not significantly affected by administration of nicotine $\left(\mathrm{F}_{(2,10)}=0.711, \mathrm{p}=0.514\right.$; see Table 3A for mean magazine latencies \pm SEM).

Correction Trials. Administration of nicotine did not significantly affect the number of correction trials committed by mice in the PAL task $\left(\mathrm{F}_{(2,10)}=1.274, \mathrm{p}=0.322\right.$; see Table $3 \mathbf{A}$ for mean number of correction trials \pm SEM).

\section{Discussion}

The present investigation sought to provide a test of utility of a mouse version of the touchscreen PAL task designed for rodents by Talpos et al. (2009) and used previously by Bartko et al. (2011). Additionally, it intended to help determine the role that the nicotinic cholinergic system plays in the ability to perform a task involving paired-associate learning, since to date, no study has presented comprehensive data on the effects of nicotinic manipulation on an appetitive rodent PAL paradigm. These goals were achieved through training mice to specified criteria of performance on the PAL task and subsequently attempting to demonstrate bidirectional modification of PAL performance via manipulation of the nicotinic cholinergic system receptors.

Bidirectional modification was hypothesized to occur from administration of the agonist nicotine (an expected improvement effect on PAL performance) and antagonist mecamylamine (an expected impairment on PAL performance). Results of this study have added to the validation of this task for pharmacological investigations and assessed the role of the central cholinergic system, particularly the nicotinic system, in mediating PAL performance. As a task that replicates the one designed for human assessment as part of the Cambridge Neuropsychological Test Automated Battery (CANTAB), the validation provided through the current investigation allows for comparison beyond rodent species (Fray et al. 1997). With this, further pharmacological investigation is possible within the mouse PAL task, with the intent of discovering those drugs that could alter performance in humans in a parallel fashion. As a task that is sensitive to those with Alzheimer's disease and Mild Cognitive Impairment, pharmacological assessment in mouse PAL has clinical significance in future drug development for diseases like Alzheimer's disease (Junkkila et al. 2012).

\section{Nature of the nicotinic manipulation effects}

A great deal of evidence within previous literature has indicated a facilitative effect of nicotine on various learning and memory performance paradigms (Levin et al. 2006; Melichercik et al. 2012). In an analysis of the effect of nicotine on an object recognition task and spatial recognition task, Melichercik et al. (2012) demonstrated an enhanced memory effect in rats upon administration of nicotine. Furthermore, Sharifzadeh et al. (2005) showed that nicotine could reverse spatial memory impairments in rats. Despite these findings, a similar effect was not seen in this study. The administration of nicotine (in both drug study A and C) at any dose in the mice sample did not significantly enhance performance on the PAL task.

Although much research has demonstrated a facilitative effect on memory from nicotine, there are also contrasting findings (Levin et al. 2006). In humans, Sahakian and Coull (1994) observed a lack of drug effect of nicotine on mnemonic memory ability, but observed nicotine-based improvements in attentional function in the same subjects. Using a group of individuals with Alzheimer's disease, White and Levin (1999) had the rationale that these patients, having lost nicotinic receptors (a product of the disease's progression), would exhibit improved memory effects upon receiving nicotine administration. Interestingly, they did not detect significant nicotine-induced effects on memory in Alzheimer's disease patients, but instead observed an improved attentional performance and faster reaction time. Finally, Sahakian et al. (1989) reported improvement in attention as measured by the working memory-based delayed response-matching task, when nicotine was administered to Alzheimer's disease subjects. Within these investigations, it appears as though nicotinic facilitative effects might be specific to tasks that are attentionally taxing. This possibility is in agreement with Warburton and Rusted (1993), who explain that nicotine's effects are most often seen in tasks that have a large attentional load, with improvement from the drug being seen in enhanced speed of processing. Stimulation of nicotinic cholinergic neurons may have a nonspecific way of providing more attentional resources. More specifically, this might involve performance enhancement on tasks that require focused attention and are speed-dependent, while not improving performance on tasks that require access to retrieval of well-learned information or semantic memory (Newhouse et al. 2001).

The PAL task variables of correct and incorrect reaction time are noteworthy in the discussion of attention. Reaction time is an effective measure used in investigations of attentional ability (de Bruin et al. 2006). In this study, it was observed that a significant effect of mecamylamine administration on both correct and incorrect affected reaction times. In particular, the mean reaction time in both situations (correct and incorrect) was significantly highest at the highest dose administered $(3.0 \mathrm{mg} / \mathrm{kg})$ than for lower dosages, with the shortest response times for both correct and incorrect trials having occurred in the no-drug saline condition. This suggests that blocking nicotinic cholinergic receptors has an impairment effect upon attention, thereby increasing the time required by the mice to make a selection. This impairment in attentional ability by administration of 


\section{Effects of nicotinic cholinergic system manipulations of PAL in mice (Milanovic)}

nicotinic receptor antagonist complements such findings as described above that administration of nicotine improves attention (Sahakian et al. 1989; White and Levin 1999).

From the results that indicated no effect of nicotine on accuracy or attention but an effect of mecamylamine on attention, the possibility emerges that within the domain of a paired-associate learning task, the nicotinic system functions at an optimal rate (i.e., cannot be improved through drug administration), but can be impaired via drug administration which blocks nicotinic cholinergic receptor function. As such, perhaps bidirectional modification is not an effect that is clearly demonstrable through nicotinic receptor manipulation in paired-associate learning; rather, modification may be specific to only the direction of impairment, with the effect being particular to attentional ability.

A final discussion on the nature of cholinergic effects on paired-associate learning performance is warranted. Bartko et al. (2011), who provided an initial investigation into cholinergic effects on rodent performance of the automated PAL task, observed that administration of donepezil, an acetylcholinesterase inhibitor and common drug treatment for Alzheimer's disease, improved performance on the task. Given that donepezil is an acetylcholinesterase inhibitor (which increases the level of acetylcholine in the central nervous system), the cholinergic effects they observed may have been muscarinic or nicotinic-based. As such, the present investigation has provided some clarity regarding the nature of this effect of donepezil. Since nicotine did not enhance PAL performance of the mice, this provides indication and evidence in support of donepezil primarily acting though the muscarinic as opposed to nicotinic system.

\section{Effects of drugs on perseverative responding}

Within the mecamylamine drug study, but not nicotine drug studies, there was an observed significant effect of the drug on a number of correction trials. Specifically, there was a significant difference between the highest $(3.0 \mathrm{mg} / \mathrm{kg})$ and lowest $(0.3 \mathrm{mg} / \mathrm{kg})$ doses of mecamylamine. The highest dose resulted in an increased number of correction trials needed. As a measure of perseverative responding and decision-making, this indicates that the highest dose of mecamylamine may have caused the mice to perseverate more frequently in their responses, responding incorrectly more often. This might be an indication of an impairment effect on PAL task performance in a dose-dependent manner, whereby the highest dose caused perseverative memory impairment. This provides some additional support to the proposal that paired-associate learning may be impaired through antagonism of the nicotinic cholinergic system, but not improved through agonism given that nicotine did not have a significant effect on decreasing the number of correction trials when all doses were compared.

\section{Effects of drugs on magazine latency}

Mecamylamine had a significant effect on magazine latency, with significantly higher latency at the highest dose compared to the three other dose conditions. This is similar to the findings of Bartko et al. (2011), who found that the muscarinic antagonists scopolamine and dicyclomine had significant effects on magazine latency, with significantly increasing latency times as dosage of antagonist increased. These results, as Bartko et al. (2011) explained, remain unclear. Perhaps the slower reaction time in obtaining the reward was a secondary effect to the primary effect of the drug on cognition (most likely the attentional effect, as currently proposed), resulting somehow in a lack of motivation to retrieve the milkshake. Or perhaps, if mecamylamine does indeed decrease attentional focus, such reduced attention may make the mice slower in retrievingtheir reward or in recognizizg the availability of the reward upon making a correct paired-associate response.

\section{Factors deserving consideration}

Newhouse et al. (2001) have proposed a general scheme in an attempt to explain nicotinic effects on learning and memory. It entails nicotinic system stimulation influencing or modulating a broad range of neurotransmitter systems, thus enhancing the performance of attentional systems and having a resultant effect of improvement in learning and memory. In particular, they suggest that nicotinic stimulation increases the activity of attentional systems, or improves discriminatory or inhibitory functioning, leading to improvements in working memory due to the increased attentional resources. In support of this, Levin (2002) explains that nicotine-induced cognitive improvement in rats is most consistently observed in tasks of working memory. Importantly, Newhouse et al. (2001) note that their proposed scheme pertains to the acquisition side of information processing. The present investigation focused on a PAL task to which the mice were pre-trained (i.e., had already acquired the task) prior to examination of drug effects on performance accuracy. In this case, the task was neither predominantly attentional-based nor an assessment of acquisition; thus, according to Newhouse et al. (2001), nicotine may have been unlikely to enhance performance. Indeed, such a result was observed, and the scheme certainly is noteworthy in the current reasoning for why bidirectional modification on the PAL task was not observed through manipulation of the nicotinic cholinergic system.

Another factor worthy of consideration is that drugs are typically given systemically, as in this study. Despite this, neurotransmitters act in anatomically precise locations (Levin et al. 2006). Drugs distribute over wide areas of the body and may activate complex systems that have receptors in various areas specialized for specific functions. As such, the sum effect of a systemically-delivered drug might be obscured by its actions on more than one system. The nicotinic system is both widespread and diverse within the central nervous system, thus providing reason to postulate that nicotinic receptors in different locations could play differing roles in neurobehavioral function (Levin et al. 2006). Perhaps some receptors work in a positive manner, having improvement effects on cognition while the same 
receptors in other brain areas may work in a negative manner upon cognition. Indeed, as demonstrated by Addy et al. (2003), nicotine agonist effects in the ventral hippocampus and basolateral amygdala appear to be opposite to the effects in the mediodorsal thalamic nucleus within working memory function. This indicates that systemic administration of nicotine (and perhaps other nicotinic agonists) could have a mutually-attenuating effect on cognitive and memory ability (Levin and Torry 1996), which could help explain why the present investigation demonstrated no enhancement effect of nicotine on PAL performance in mice.

Future research attempting to demonstrate bidirectional modification should assess the effect of nicotine and mecamylamine on PAL performance through intrahippocampal and intra-perirhinal cortex administration. These are the areas specifically implicated in visuospatial paired-associate learning, as used in the present investigation. This form of administration would help to eliminate mutually-attenuating effects from activation of oppositely acting nicotinic receptors from systemic administration. Furthermore, it would permit for the observation of whether paired-associate learning can be enhanced through nicotinic activation and impaired through nicotinic deactivation when the areas of the brain that subserve the ability to perform this task are specifically targeted.

\section{Future directions and importance}

In previous rodent studies on PAL task performance, data on cholinergic effects have only been provided for the effects of muscarinic and nicotinic antagonism, with the muscarinic receptor antagonist scopolamine impairing rodent performance on the PAL automated task and the nicotinic antagonist mecamylamine not impairing task performance (Talpos et al. 2009; Bartko et al. 2011). While the current study investigated effects of both nicotinic agonism and antagonism on performance, a comprehensive assessment such as this has not been conducted within the muscarinic cholinergic system. As such, it is unknown whether similar results would be produced in attempted bidirectional modification of performance in the PAL task with the muscarinic system as a target. The investigation will need to be conducted on this novel tool of paired-associate learning within a rodent sample to determine whether the effects observed here are specific to the nicotinic system.

Future investigations intending to contribute to this line of research performed using the mouse model of the PAL task might consider assessing a larger sample of mice than used here to enhance power in detecting drug effects. Additionally, it is possible that higher doses than used here would result in significant drug effects. Perhaps the dosages selected in the present study were not high enough to elicit detectable bidirectional modification of PAL performance. Future research might increase the dosage values used; however, it should be noted that seizure activity was observed in mice receiving the highest dosage of nicotine $(1.0 \mathrm{mg} / \mathrm{kg})$ used in drug study C.
The present study provides one of the first investigations into the touchscreen automated mouse PAL task, helping to establish it as a valuable tool for pharmacological investigations in mice. As suggested by Bartko et al. (2011), a touchscreen apparatus such as this provides a useful mode of testing cognitive ability and has the potential to be used as part of a battery of cognitive tests for applications in drug discovery. Furthermore, this investigation has provided insight into the role that the nicotinic system plays in the capacity to perform a task of paired-associate learning, which has implications and applications within the medical world. Specifically, these findings pertain to learning and memory deficits as seen in patients diagnosed with Alzheimer's disease and Mild Cognitive Impairment and also may be considered in future drug investigations aimed at contributing to clinical knowledge and therapeutic availabilities.

\section{Acknowledgements}

This work was supported by a grant from the Natural Sciences and Engineering Research Council of Canada (NSERC).

\section{References}

Addy N, Nakajama A, Levin E. 2003. Nicotinic mechanisms of memory: Effects of acute local DHbE and MLA infusions in the basolateral amygdala. Cognitive Brain Research. 16:51-57.

Aggleton JP, Hunt PR, Rawlins JNP. 1986. The effects of hippocampal lesions upon spatial and non-spatial tests of working memory. Behavioural Brain Research. 19:133-146.

Augzzi A, Brander S, Sure U, Ruedi D, Isenmann S. 1994. Transgenic and knock-out mice: Models of neurological disease. Brain Pathology. 4:3-20.

Alexander GE, DeLong MR, Strick PL. 1986. Parallel organization of functionally segregated circuits linking basal ganglia and cortex. Annual Review of Neuroscience. 9: 357-381.

Bartholemew HJD, Lindner MD, Hogan JB, Jones KM, Markus EJ. 2009. Scopolamine induced deficits in a battery of rat cognitive tests: Comparisons of sensitivity and specificity. Behavioural Pharmacology. 20:237-251.

Bartko SJ, Vendrell I, Saksida LM, Bussey TJ. 2011. A computer-automated touchscreen paired-associates learning (PAL) task for mice: Impairments following administration of scopolamine or 


\section{Effects of nicotinic cholinergic system manipulations of PAL in mice (Milanovic)}

dicyclomine and improvements following donepezil. Psychopharmacology. 214:537-548.

Bartus RT. 1978. Evidence for a direct cholinergic involvement in the scopolamine-induced amnesia in monkeys: Effects of concurrent administration of physostigmine and methylphenidate with scopolamine. Pharmacology Biochemistry and Behaviour. 9:833-836.

Bartus RT, Johnson HR. 1976. Short-term memory in the rhesus monkey: Disruption from the anti-cholinergic scopolamine. Pharmacology Biochemistry and Behaviour. 5:31-39.

Biala G, Kruk M. 2008. Cannabinoid receptor ligands suppress memory-related effects of nicotine in the elevated plus maze test in mice. Behavioural Brain Research. 192(2):198-202.

Blackwell AD, Sahakian BJ, Vesey R, Semple JM, Robbins JM, Robbins TW, Hodges JR. 2004. Detecting dementia: Novel neuropsychological markers of preclinical Alzheimer's disease. Dementia and Geriatric Cognitive Disorders. 17:42-48.

Braak H, Braak E. 1997. Frequency of stages of Alzheimerrelated lesions in different age categories. Neurobiology of Aging. 18:351-357.

Bussey TJ, Saksida LM, Rothnlat LA. 2001. Discrimination of computer-graphic stimuli by mice: A method for the behavioral characterization of transgenic and gene-knockout models. Behavioral Neuroscience. 115(4):957-960.

Carli M, Robbins TW, Evenden JL, Everitt BJ. 1983. Effects of lesions to ascending noradrenergic neurons on performance of a 5-choice serial reaction task in rats: Implications for theories of dorsal noradrenergic bundle function based on selective attention and arousal. Behavioral Brain Research. 9:361-380.

Caulfield MP. 1993. Muscarinic receptors - characterization, coupling and function. Pharmacology \& Therapy. 58:319-379.

Clarke PBS, Chaudieu I, el-Bizri H, Boksa P, Quick M, Esplin BA, Capek R. 1994. The pharmacology of the nicotinic antagonist, chlorisondamine, investigated in rat brain and autonomic ganglion. British Journal of Pharmacology. 111:397-405.
Clelland CD, Choi M, Romberh C, Clemenson Jr GD, Fragniere A, Tyers P, Jessberger S, Saksida LM, Barker RA, Gage FH, Bussey TJ. 2009. A functional role for adult hippocampal neurogenesis in spatial pattern separation. Science. 325(5937):210-213.

Colquohoun LM, Patrick JW. 1997. Pharmacology of neuronal nicotinic acetylcholine receptor subtypes. Advanced Pharmacology. 39:191-200.

De Bruin NM, Fransen F, Duytschaever H, Grantham C, Megens AA. 2006. Attentional performance of (C57BL/6Jx129Sv) F2 mice in the five-choice serial reaction time task. Physiology \& Behaviour. 89:692-703.

Domjan M, Purdy JE. 1995. Animal research in psychology: More than meets the eye of the general psychology student. American Psychologist. 50(7):496-503.

Drachman DA. 1977. Memory and cognitive function in man: Does the cholinergic system have a specific role? Neurology. 27:783-790.

Drachman DA, Leavitt J. 1974. Human memory and the cholinergic system. A relationship to aging? Archives of Neurology. 30:113-121.

Forwood SE, Winters BD, Bussey TJ. 2005. Hippocampal lesions that abolish spatial maze performance spare object recognition memory at delays of up to 48 hours. Hippocampus. 15:347-355.

Fray PJ, Robbins TW, Sahakian BJ. 1997. Neuropsychiatric applications of CANTAB. International Journal of Geriatric Psychiatry. 11:329-336.

Frumin MJ, Herekar VR, Jarvik ME. 1976. Amnesic actions of diazepam and scopolamine in man. Anestesiology. 45:406-412.

Graef S, Schonknecht P, Sabri O, Hegerl U. 2011. Cholinergic receptor subtypes and their role in cognition, emotion, and vigilance control: An overview of preclinical and clinical findings. Psychopharmacology. 215:205-229.

Hagan JJ, Jansen JHM, Broekkamp CLE. 1987. Blockade of spatial learning by the $\mathrm{m} 1$ muscarinic antagonist pirenzipine. Psychopharmacology. 93: 470-476.

Hahn B, Shoaib M, Stolerman IP. 2002. Nicotine-induced enhancement of attention in the five-choice serial reaction time task: The influence of task demands. Psychopharmacology. 162:129-137. 
James JR, Nordberg A. 1995. Genetic and environmental aspects of the role of nicotinic receptors in neurodegenerative disorders: Emphasis on Alzheimer's disease and Parkinson's disease. Behavioural Genetics. 25:149-159.

Junkkila J, Oja S, Laine M, Karrasch M. 2012. Applicability of the CANTAB-PAL computerized memory test in identifying amnestic mild cognitive impairment and Alzheimer's Disease. Dementia and Geriatric Cognitive Disorders. 34(2):83-89.

Katner SN, Davis SA, Kirsten AJ, Taffe MA. 2004. Effects of nicotine and mecamylamine on cognition in rhesus monkeys. Psychopharmacology. 175: $225-240$.

Kim J, Levin E. 1996. Nicotinic, muscarinic and dopaminergic actions in the ventral hippocampus and the nucleus accumbens: Effects on spatial working memory in rats. Brain Research. 725: 231-240.

Levin ED. 2002. Nicotinic receptor subtypes and cognitive function. Journal of Neurobiology. 53:633-640.

Levin ED, McClernon FJ, Rezvani AH. 2006. Nicotinic effects on cognitive function: Behavioral characterization, pharmacological specification, and anatomic localization. Psychopharmacology. 184:523-539.

Levin ED, Torry D. 1996. Acute and chronic nicotine effects on working memory in aged rats. Psychopharmacology. 123:88-97.

Melichercik AM, Elliott KS, Bianchi C, Ernst SM, Winters BD. 2012. Nicotinic receptor activation in perirhinal cortex and hippocampus enhances object memory in rats. Neuropharmacology. 62:2096-2105.

Morton AJ, Skillings E, Bussey TJ, Saksida LM. 2006. Measuring cognitive deficits in disabled mice using an automated interactive touchscreen system. Nature Methods. 3:767.

Mumby DG, Pinel JPJ. 1994. Rhinal cortex lesions and object recognition in rats. Behavioural Neuroscience. 108(1):11-18.

Newhouse PA, Potter A, Kelton M, Corwin J. 2001. Nicotinic treatment of Alzheimer's disease. Biological Psychiatry. 49:268-278.

O'Keefe J, Nadel L. 1978. The hippocampus as a cognitive map. Oxford: Clarendon Press. 570 p.
Revised Statues of Ontario. 1990. Animals for Research Act (Ontario) Ch. A.22. Service Ontario.

Rodewald HK. 1973. Application of Bowler's One-Element Model to paired-associate learning by pigeons. Journal of the Experimental Analysis of Behavior. 19(2):219-223.

Sahakian BJ, Coull JT. 1994. Nicotine and tetrahydroaminoacradine: Evidence for improved attention in patients with dementia of the Alzheimer type. Drug Development Research. 31:80-88.

Sahakian BJ, Morris RG, Evenden JL, Heald A, Levy R, Philpot M, Robbins TW. 1989. A comparative study of visuospatial memory and learning in Alzheimertype dementia and Parkinson's disease. Brain III. 3:695-718.

Sharifzadeh M, Tavasoli M, Naghdi N, Ghanbari A, Amini M, Roghani A. 2005. Post-training intrahippocampal infusion of nicotine prevents spatial memory retention deficits induced by the cyclo-oxygenase-2-specific inhibitor celecoxib in rats. Journal of Neurochemistry. 95:1078-1090.

Snaedal J, Johannesson T, Jonsson JE, Gylfadottir G. 1996. The effects of nicotine in dermal plaster on cognitive functions in patients with Alzheimer's disease. Dementia. 7:47-52.

Swainson R, Hodges JR, Galton CJ, Semple J, Michael A, Dunn BD, Iddon JL, Robbins TW, Sahakian BJ. 2001. Early detection and differential diagnosis of Alzheimer's disease and depression with neuropsychological tasks. Demential and Geriatric Cognitive Disorders. 12(4):265-280.

Talpos JC, Winters BD, Dias R, Saksida LM, Bussey TJ. 2009. A novel touchscreen-automated pairedassociate learning (PAL) task sensitive to pharmacological manipulation of the hippocampus: A translational rodent model of cognitive impairments in neurodegenerative disease. Psychopharmacology. 205:157-168.

Terry AV, Buccafusco JJ, Jackson WJ, Zagrodnik S, EvansMartin FF, Decker MW. 1996. Effects of stimulation or blockade of central nicotiniccholinergic receptors on performance of a novel version of the rat stimulus discrimination task. Psychopharmacology (Berl). 123:172-181.

Warburton DM, Rusted JM. 1993. Cholinergic control of cognitive resources. Neuropsychobiology. 28: 43-46. 
White HK, Levin ED. 1999. Four-week nicotine skin patch treatment effects on cognitive performance in Alzheimer's disease. Psychopharmacology. 143:158-165.

Winters BD, Bussey TJ. 2005. Transient inactivation of perirhinal cortex disrupts encoding, retrieval, and consolidation of object recognition memory. Journal of Neuroscience. 25(1):52-61.
Young JW, Finlayson K, Spratt C, Marston HM, Crawford N, Kelly JS, Sharkey J. 2004. Nicotine improves sustained attention in mice: Evidence for involvement of the alpha7 nicotinic acetylcholine receptor. Neuropsychopharmacology. 29:891-900. 


\section{Effects of nicotinic cholinergic system manipulations of PAL in mice (Milanovic)}

\section{Supplementary Information}

\section{Subjects}

Mice were pair-housed with corncob bedding and environmental enrichment in polyethylene cages $(16 \mathrm{~cm} \times 12 \mathrm{~cm} \times 26 \mathrm{~cm})$, in a room with a 12-hour light/dark cycle (lights on at 8:00 p.m.). All behavioural testing was conducted during the dark phase of the cycle. Mice received rodent chow (Tekland Global 14\% Protein Rodent Maintenance Diet, Harlan Teklad, WI) and their diets were restricted to maintain their weights at $85 \%$ of free feeding body weight during behavioural testing. Water was available ad libitum throughout the experiment. All experimentation was conducted in accordance with the Animals for Research Act (Ontario) and its regulations (Revised Statutes of Ontario, 1990, Chapter A.22).

\section{Apparatus}

This apparatus was comprised of a testing chamber within a sound- and light-attenuating box $(40 \mathrm{~cm} \times 34 \mathrm{~cm} \times 42 \mathrm{~cm}$; Lafayette Instrument Company Inc., Indiana, US). The inner operant chamber consisted of a metal frame and stainless steel grid floor. The box was equipped with a fan to ventilate and mask extraneous noise, and a milkshake receptacle (magazine) that was illuminated by a 3-W light bulb and fitted with a photocell head entry detector attached to the milkshake dispenser tube. This tube connected to an extraneous reservoir of milkshake (reward). The behavioural testing program was controlled by computer software program Animal Behavior Environment Test System (ABET II) (Lafayette Instrument Company Inc., Indiana, US).

\section{Pretraining}

The mice underwent one week of handling prior to training to become accustomed to handling by the researcher. Their body weights were closely monitored throughout the duration of the experimental process. The mice were placed in the chamber for two daily 15 -minute habituation sessions during which the house light remained on and milkshake was placed in the magazine. Following this habituation stage, the mice were trained to collect milkshake delivered under a variable interval 30 -second schedule, coinciding with magazine illumination and a tone. Training stimuli (40 stimuli varying in shape, pattern, and brightness) were presented one per trial in one of the three response windows. Delivery of a drop of milkshake immediately followed stimulus offset. If the mouse touched the stimulus, the stimulus disappeared immediately and the mouse was rewarded with the tone, magazine light, and milkshake.

The next training session required the mouse to respond to the stimulus presented on the touchscreen to receive milkshake. A training stimulus was shown in one of the three response windows on each trial, and remained there until the mouse responded by nose-poking it. The mouse was then rewarded with a drop of milkshake, tone, and illumination of the magazine light. After a 20-second intertrial interval (ITI), the next trial commenced with presentation of a new stimulus. Once the mouse successfully completed 30 trials in a 60 -minute session, it entered the next training stage in which it was required to initiate trials. After collecting the reward following the response to a stimulus and leaving the reward magazine, the mouse had to poke its head in the magazine to cause the next stimulus to be presented in one of the screen windows. To pass this stage the mouse needed to successfully initiate and complete 30 trials in one hour.

In the final training stage, a cue to signal incorrect responses (responding to a window in which no stimulus had been displayed) was presented, and a correction procedure occurred. Correct responses to the window in which the stimulus was presented were followed by stimulus offset, and presentation of a drop of milkshake, tone, and illuminated food magazine (followed by a 20-second ITI). Following incorrect responses, the house light extinguished, the stimulus disappeared, and a 5-second time-out period occurred, followed by a 20second ITI. A correction procedure was then implemented, whereby the trial was repeated until the mouse made the correct choice. Once the mouse could complete this stage at $70 \%$ correct (this measure pertained to non-correction trials only, not correction trials) over two consecutive sessions, PAL task training began.

\section{Training of the PAL task}

At the commencement of a daily session, the mouse was required to initiate the first trial. Following this, two stimuli would appear on the screen in two of the three windows: left, middle, and right. One of these stimuli was the correct S+ while the other was the incorrect S-. There were six possible trial types and three different stimuli used (flower, plane, spider). Within the six trial types, the flower was rewarded only when presented in the left screen, the plane was rewarded only when presented in the middle location, and the spider was rewarded only when presented in the right location. As such, the mouse had to learn the paired-associations of the three locations and visual stimuli. A nosepoke to the correct $\mathrm{S}+$ resulted in a milkshake reward, tone and magazine light. A nose-poke to the incorrect $\mathrm{S}$ - resulted in a 5-second timeout period, then correction procedure. If a mouse nose-poked a window in which no stimulus was presented, this was ignored. A 20-second ITI was presented between each stimulus trial. The mice were required to complete 36 trials in one hour (each trial type could occur six times and the maximum number of times a trial could occur consecutively was twice). 